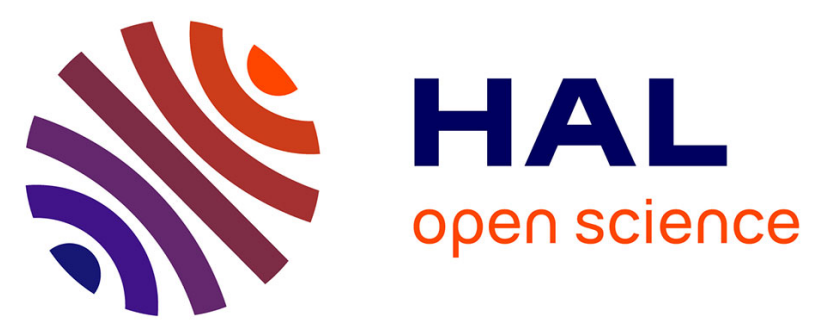

\title{
Low Field Laser Ionization of Argon Clusters: The Remarkable Fragmentation Dynamics of Doubly Ionized Clusters
}

\author{
Lionel Poisson, Kevin Raffael, Marc-André Gaveau, Benoît Soep, Jean-Michel \\ Mestdagh, Jérémie Caillat, Richard Taïeb, Alfred Maquet
}

\section{To cite this version:}

Lionel Poisson, Kevin Raffael, Marc-André Gaveau, Benoît Soep, Jean-Michel Mestdagh, et al.. Low Field Laser Ionization of Argon Clusters: The Remarkable Fragmentation Dynamics of Doubly Ionized Clusters. Physical Review Letters, 2007, 99 (10), pp.103401. 10.1103/PhysRevLett.99.103401 . hal01955588

\section{HAL Id: hal-01955588 \\ https://hal.sorbonne-universite.fr/hal-01955588}

Submitted on 18 Dec 2018

HAL is a multi-disciplinary open access archive for the deposit and dissemination of scientific research documents, whether they are published or not. The documents may come from teaching and research institutions in France or abroad, or from public or private research centers.
L'archive ouverte pluridisciplinaire HAL, est destinée au dépôt et à la diffusion de documents scientifiques de niveau recherche, publiés ou non, émanant des établissements d'enseignement et de recherche français ou étrangers, des laboratoires publics ou privés. 


\title{
Low Field Laser Ionization of Argon Clusters: The Remarkable Fragmentation Dynamics of Doubly Ionized Clusters
}

\author{
Lionel Poisson, ${ }^{*}$ Kevin D. Raffael, Marc-André Gaveau, Benoît Soep, and Jean-Michel Mestdagh \\ Laboratoire Francis Perrin, CEA/DSM/DRECAM/SPAM-CNRS URA 2453, C.E.A. Saclay, F-91191 Gif-sur-Yvette cedex, France
}

Jérémie Caillat, Richard Taïeb, and Alfred Maquet

Laboratoire de Chimie Physique-Matière et Rayonnement, Université Pierre et Marie Curie-Paris VI, CNRS UMR 7614,

11 Rue Pierre et Marie Curie, 75231 Paris Cedex 05, France

(Received 19 March 2007; published 7 September 2007)

\begin{abstract}
We have investigated the fission following a Coulomb explosion in argon clusters (up to $\operatorname{Ar}_{800}$ ) irradiated by a femtosecond infrared laser with moderate intensity $I_{L} \approx 10^{13} \mathrm{~W} \mathrm{~cm}^{-2}$. We report the $a$ priori surprising observation of well-defined velocity distributions of the ionized fragments $\mathrm{Ar}_{n<50}^{+}$. This is interpreted by the formation of a valence shell excited charged ion, followed by relaxation, charge transfer by autoionizing collision at very short distance, and asymmetric fission.
\end{abstract}

DOI: 10.1103/PhysRevLett.99.103401

The ionization dynamics and the subsequent fragmentation of van der Waals clusters exposed to intense laser pulses is an important issue in the physics of laser-matter interactions [1]. This stems from the unique properties of clusters, at the border between the dilute and the condensed phases. Within this context, recent experiments have shown that rare gas clusters, irradiated by strong infrared (ir) laser pulses, display interesting and unexpected phenomena that differ significantly from those produced in isolated atoms. Notably, the production of fast, highly charged species and the emission of "hot" electrons was observed at rather high laser intensities, beyond $I_{L}=$ $10^{16} \mathrm{~W} \mathrm{~cm}^{-2}[2,3]$. Together, the emission of characteristic $\mathrm{x}$-ray radiations was reported at slightly lower intensities, $I_{L} \approx 10^{15} \mathrm{~W} \mathrm{~cm}^{-2}$ [4]. The dominant features of these effects can be accounted for in terms of the response of the underdense plasma created after the atoms in the clusters have been stripped of their outer electrons.

In contrast to these studies conducted in the high intensity regime, the present Letter addresses the fragmentation dynamics that results from the double ionization of medium sized argon clusters $\operatorname{Ar}_{\bar{N}}(\bar{N}$, average size $500 \lesssim \bar{N} \lesssim$ 800 ), when irradiated by ir laser pulses of "moderate" intensity, $I_{L}=3.6 \times 10^{13} \mathrm{~W} \mathrm{~cm}^{-2}$. The main findings of our study are related to the $a$ priori unexpected velocity distribution of the singly charged fragments ejected in the course of the fragmentation of laser-irradiated clusters $\operatorname{Ar}_{\bar{N}}$. The analysis of these results sheds light on the dynamics of doubly ionized clusters, in a regime that differs from previous studies performed by electron-impact ionization [5], heavy ion collision [6] and inner-shell x-ray Auger emission [7-9].

In particular, we infer that, in spite of the rather moderate intensity of the ir laser, an ionization mechanism leading to the formation of an ion pair $\mathrm{Ar}^{+}-\mathrm{Ar}^{+}$, at a short interionic distance results in Coulomb explosion followed by the cluster fission.
PACS numbers: $36.40 . \mathrm{Qv}, 32.80 . \mathrm{Dz}, 82.50 . \mathrm{Pt}, 82.53 .-\mathrm{k}$

This mechanism, which was not anticipated, is interpreted by electron recollision within the cluster. It presents some similarities with the formation of doubly ionized argon clusters induced by soft-x-ray synchrotron radiation, where the initial stage is an Auger process [7-9]. However, in those measurements, the velocity correlation observed between the small fragments is lost for $\operatorname{Ar}_{\bar{N}>60}$. Other experiments performed by electron impact [5] or heavy ions collisions [6] have evidenced other specific ionization mechanisms. In the present study, we uncover a new frag-
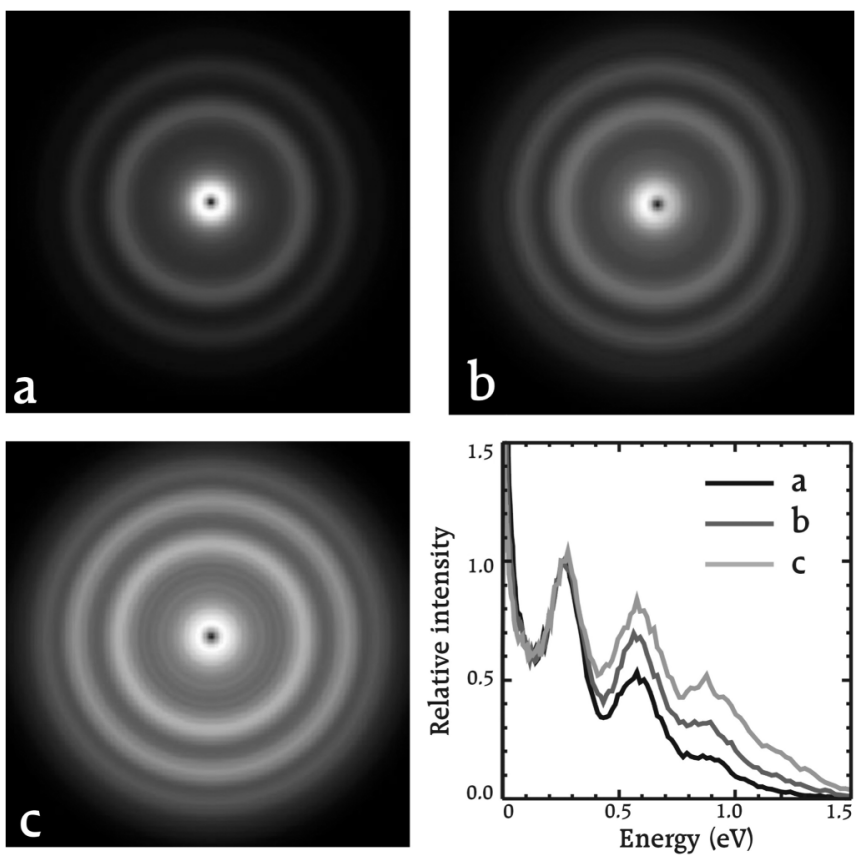

FIG. 1. Velocity distribution of $\mathrm{Ar}_{15}^{+}$for an average cluster size of (a) $\bar{N}=630$, (b) $\bar{N}=680$, (c) $\bar{N}=750$. Images are averaged over all angles, intensity at radius $r$ was multiplied by $r$ and normalized. The color map applied is linear. Curves are normalized at the peak of the first ring. 
mentation scheme for large clusters, that involves atomic outer shell ionization.

The experimental setup has been described previously [10]. High purity argon was expanded into vacuum through a pulsed $100 \mu \mathrm{m}$ nozzle. The resulting beam was extracted using a $1 \mathrm{~mm}$ skimmer. The argon backing pressure was adjusted in the range 3 to 15 bars, corresponding to the production of clusters up to $\mathrm{Ar}_{\sim 800}$, following the approximate rule of 50 atoms bar ${ }^{-1}$ [11]. The clusters are ionized by a femtosecond Ti:sapphire $(800 \mathrm{~nm}, 70 \mathrm{fs}$ full width at half maximum) laser beam from the low energy beam line of LUCA (a member of the European facility SLIC). The maximum intensity of the laser was $I_{\max }=3.6 \times$ $10^{13} \mathrm{~W} \mathrm{~cm}^{-2}$. The resulting ions were analyzed using of a velocity map imaging (VMI) device [12]. The images were collected under slicing conditions [13]. Given the size of the detector, the analysis is limited to ionic fragments with kinetic energies $\leqslant 3 \mathrm{eV}$.

In order to study ions ejected from large clusters after ionization, the signal due to $\mathrm{Ar}^{+}$and $\mathrm{Ar}_{2}^{+}$ions was not recorded although it represents about $95 \%$ of the total. Instead, we analyzed the remaining 5\% of the total signal, and cluster ions were considered from $\mathrm{Ar}_{3}^{+}$to $\mathrm{Ar}_{50}^{+}$. $\mathrm{A}$ typical result is shown in Fig. 1 for the $\mathrm{Ar}_{15}^{+}$ions. All the distributions have the same structure, namely, a central peak, close to the zero kinetic energy, surrounded by a series of well-defined rings. Remarkably, the rings display a uniform density and there is no indication of a dissymmetry along the polarization direction of the laser (parallel to the plane of the figure). We point out that, for the smaller fragments $\left(\mathrm{Ar}_{3}^{+}\right.$and $\left.\mathrm{Ar}_{4}^{+}\right)$the rings are broader and overlap partially. We shall return to this point later.

The central peak is attributed to the single ionization of the clusters present in the beam. Since the sudden formation of a charge within the cluster creates a nonequilibrium situation, a vibrational relaxation follows which promotes evaporation and fragmentation [14]. Hence, the shape of this peak corresponds to the distribution of the recoil velocities of the evaporated $\mathrm{Ar}_{n}^{+}$ions $\left(\mathrm{Ar}_{15}^{+}\right.$in Fig. 1). This point will be the subject of a forthcoming study and is not considered further.

The ring structure, which is typical of all the ion images obtained in the present study, can be related to the formation of multicharged clusters, from which the singly charged fragments originate upon Coulomb explosion. One observes a series of two rings in panel (a) of Fig. 1 and three rings in panels (b) and (c) when the argon backing pressure, i.e., the average cluster size, increases. The latter three rings are associated with energies of $0.29 \pm$ $0.01,0.590 \pm 0.005$, and $0.90 \pm 0.01 \mathrm{eV}$, respectively. These energies were derived from a fit of the rings with Gaussian functions. An important feature appears in Fig. 1 for the $\mathrm{Ar}_{15}^{+}$ion signal: the energy and width of the rings do not depend on the average size of the neutral parent clusters; only the relative magnitude of the ring changes with it.
Outer rings are brighter at larger sizes of the neutral clusters. The same behavior is found in all the cluster ion signals that were recorded.

Another interesting feature is that the ring structure does not depend significantly on the laser intensity, in the range considered here $\left(I_{L}<3.6 \times 10^{13} \mathrm{~W} \mathrm{~cm}^{-2}\right)$. For example, when dividing the laser intensity by a factor of 3 in the experiment reported in Fig. 1, the total ion signal was divided by a factor of about 100 , whereas the ring energies remained unchanged and the relative intensity of the rings was changed only very weakly. The same structures are also observed when using the second harmonic of the laser $\left(400 \mathrm{~nm}, I_{L} \leq 5.0 \times 10^{13} \mathrm{~W} \mathrm{~cm}^{-2}\right)$.

We note that the energies of the rings observed in Fig. 1 follow approximately a progression given by $E_{p+1}-E_{p} \sim$ $0.29 \mathrm{eV}$, where $p$ denotes the ring number. This general result is illustrated in panel (a) of Fig. 2 showing the energy of the first four rings of the ion images taken for $\operatorname{Ar}_{n}^{+}(n \in$ $[6,50])$. Panel (b) displays as the function of fragment ion $\mathrm{Ar}_{n}^{+}$, the same information normalized with respect to the first ring. The relative ring energies are in the ratio 1:2:3:4 when $n \gtrsim 20$.

To address the question of the ionization dynamics, we note first that the cluster ions corresponding to the outer rings are fairly energetic, up to $1.4 \mathrm{eV}$ for the fourth ring in the energy spectrum of $\mathrm{Ar}_{10}^{+}$. This suggests that Coulomb explosion takes place, hence implying that multiple ionization is an active process in spite of the moderate laser intensity. Within this framework, three scenarios based on direct cluster double ionization can be invoked. One [scenario (i)], is associated with the initial ionization of two atoms randomly distributed within the cluster. Another one [scenario (ii)], implies the ionization of two adjacent atoms, while a third [scenario (iii)] implies the double ionization of a single Ar atom. An alternative one [scenario
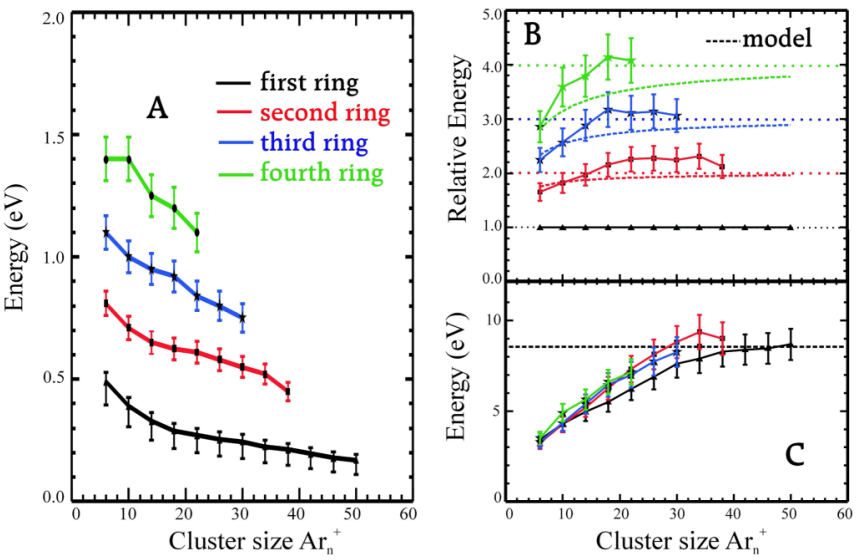

FIG. 2 (color online). Energy evolution for an average cluster size of $\mathrm{Ar}_{650}$. Rings of an overly weak intensity are not reported. (a) Ring energies as a function of the fragment size. (b) Energy ratios relative to the first ring. (c) Total recoil energy issued from the model (see text). 
(iv)] implies an indirect double ionization. The initial stage involves the formation of an excited $\mathrm{Ar}^{+*}$ ion which triggers the formation of an ion pair $\mathrm{Ar}^{+}-\mathrm{Ar}^{+}$separated by a short distance.

It turns out that the initial ionization of two atoms randomly distributed [scenario (i)] is excluded because the recoil energy of the fragments would decrease when the average neutral cluster size increases. Indeed, the average distance $r$ between two atoms in the cluster is given by the pair correlation function $4 \pi r^{2} g(r)$. When transformed into Coulomb energies, such a distribution of distances leads to a broad range of recoil energies after Coulomb explosion, as confirmed by a histogram calculation based on Lennard-Jones-type cluster geometry [15]. For example, $A_{800}$ (respectively, $A_{500}$ ) clusters would show distributions peaking at a total repulsion energy of $0.55 \mathrm{eV}$ (respectively, $0.62 \mathrm{eV}$ ), with a width of $0.4 \mathrm{eV}$ (respectively, $0.5 \mathrm{eV}$ ). As these energies are shared between the fragments, this is neither consistent with the measured values, nor with the stability of the ring position when changing the average cluster size.

Scenario (ii) implies the single ionization of two nearby atoms, aligned along the polarization direction of the laser field. It is expected that this process is a priori favored as compared to the one involved in scenario (i), since, within a cluster, two neighboring atoms are more easily ionized than the isolated ones. However, the potential energy of two ions located at the interatomic distance between two neutral Ar would lead to lower kinetic energy distributions of the fragments than observed in the present work. Moreover, it is anticipated that such a process would induce a strong polarization dependence in the angular distribution of the fragments. This is not observed experimentally. The angular distribution of the ionized fragments is found, indeed, to be isotropic (anisotropy parameter $\beta \simeq 0$ within noise) .

Regarding scenario (iii), despite a higher ionization potential of $\mathrm{Ar}^{+}$versus $\mathrm{Ar}(27.63 \mathrm{eV}$ versus $15.76 \mathrm{eV})$, it appears that the double ionization of a single atom is likely to contribute also because of the recollision of the outgoing electron with the remaining $\mathrm{Ar}^{+}$ion during the laser pulse. The ratio between the double- versus single-ionization probabilities of argon is almost constant, with $\left(P^{(2+)} / P^{(+)}\right) \geqslant 10^{-3}$, over the intensity range of the ir laser considered here [16]. Assuming that the ionization probability is about unity at the saturation threshold, the single-ionization probability is estimated to $P^{(+)} \approx 10^{-6}$, whereas a conservative estimate of the double ionization probability is $P^{(2+)} \approx 10^{-9}$. Hence, the ionization probability for two separate atoms (i) within $\mathrm{Ar}_{N}$ can be estimated to be $P_{(\mathrm{i})} \approx C_{2}^{N} \times\left(10^{-6}\right)^{2}$ while the probability of double ionization of an isolated atom (iii) is $P_{(\text {iii) }} \approx N \times$ $10^{-9}$. The ratio $P_{(\mathrm{i})} / P_{(\mathrm{iii})} \approx(N / 2) \times 10^{-3}$ suggests that both scenarios are expected to contribute to the ionization for the cluster size range investigated here, in spite of the strict energetic considerations. With a similar statistical approach, we conclude that it is not necessary to consider the triple ionization (and beyond).

Within the framework of the scenario (iii), the laser would double ionize initially a single atom of the cluster. This would form a localized charged center that favors collisions between the doubly charged center and neighboring argon atoms, thus inducing a charge transfer. Such a process has been documented in the case of argon dimers $\mathrm{Ar}_{2}$, when one of the atoms experiences an Auger transition $[9,17]$. The charge transfer would occur at much shorter distances than the equilibrium distance in neutral $\mathrm{Ar}_{2}[17,18]$. In the present case, ground state $\mathrm{Ar}^{2+}$ is likely to be formed and no curve crossing exists that will induce the $\mathrm{Ar}^{2+}+\mathrm{Ar} \rightarrow \mathrm{Ar}^{+}+\mathrm{Ar}^{+}$charge transfer [17,19]. For this reason, we prefer the alternative interpretation [scenario (iv)] where an electronically excited $\mathrm{Ar}^{+*}$ ion is formed by the same recollision mechanism evoked in scenario (iii). It is expected that this nonsequential mechanism is more efficient for excitation than for ionization. Hence, according to the former statistical considerations, scenario (iv) is likely to be the dominant one. Because of its high ionization potential, $\mathrm{Ar}^{+}$has excited states high enough to allow the $\mathrm{Ar}^{+*}+\mathrm{Ar} \rightarrow \mathrm{Ar}^{+}+\mathrm{Ar}^{+}+e^{-}$process [Fig. 3(a)]. Preliminary ab initio calculations [20] indicate that this transfer should occur at a significantly shorter distance, $r_{\text {crit }}$, than the Ar-Ar distance in the neutral species. We note here that the excitation process invoked involves mostly $m_{l}=0$ electrons, that limits the number of $\mathrm{Ar}^{+}$Rydberg states to those correlating with the ${ }^{1} D$ and ${ }^{1} S$ excited states of $\mathrm{Ar}^{2+}$.

Coulomb explosion results from charge transfer [Fig. 3(c)]. It generates singly ionized fragments: $\mathrm{Ar}_{N}^{2+} \rightarrow$ $\mathrm{Ar}_{p}^{+}+\mathrm{Ar}_{n}^{+}+\mathrm{Ar}_{N-(n+p)}$. The available energy for the dissociation as $\mathrm{Ar}_{p}^{+}+\mathrm{Ar}_{n}^{+}$is partly due to the Coulomb energy $E_{c}=e^{2} /\left(4 \pi \epsilon_{0} r_{\text {crit }}\right)$ and partly to the energy corresponding to the structural rearrangement [Fig. 3(b)] for the system to reach $r_{\text {crit }}$. The kinetic energy released $(E)$ after dissociation is shared between both partners according to the values of $n$ and $p$ [Fig. 3(d)]. The one of $\mathrm{Ar}_{n}^{+}$can be modeled by $E_{n}=p /(n+p) E$ (momentum conservation), neglecting the fission energy as compared to the recoil energy. Accordingly, the $p$ th ring for the fragments

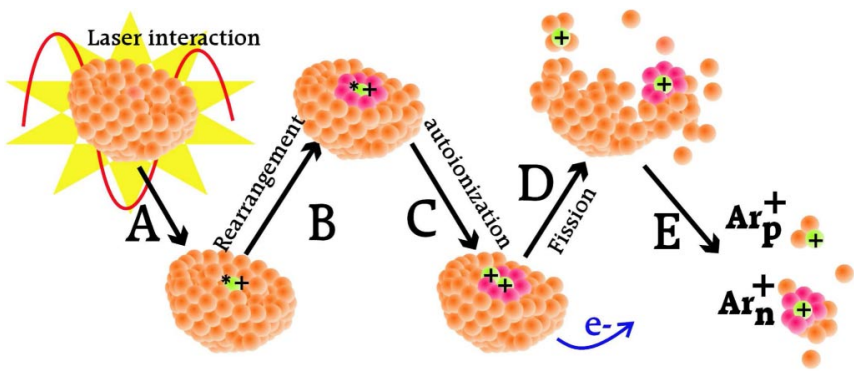

FIG. 3 (color online). Dissociation channel proposed. Scenario (iv). 
$\operatorname{Ar}_{n}^{+}$with $n \geq 6$ can be associated with the recoil partner $\mathrm{Ar}_{p}^{+}$. Then the ring structure corresponds to the values $p=$ $1,2,3,4, \ldots$ This agrees reasonably well with the $n$ dependence of the ring energies reported in Fig. 2(b). Applying the model to the experimental recoil energies gives access to the total energy involved in the fragmentation [see Fig. 2(c)]. This energy should be the same whatever the size of the fragment. This is observed for $\mathrm{Ar}_{N \geqslant 25}^{+}$ and gives an energy of about $8.5 \mathrm{eV}$, suggesting $r_{\text {crit }} \simeq$ $1.7 \pm 0.2 \AA$ (with the approximation $E_{\text {tot }}=E_{c}$ ), much shorter than the distance in the neutral cluster $(3.76 \AA$ [7]). The deviation from this estimate for the smaller fragments is attributed to the evaporation following the fission of the cluster. Indeed, the observation of magic numbers for $\mathrm{Ar}_{n}^{+}$(through their relative global ring intensities) is indicative of an evaporation process subsequent to fission [Fig. 3(e)]. However, it is not important enough to scramble the ring structure. The mechanism causing the charge transfer could be of Coulombic or vibronic origin. This first one, called interatomic Coulombic decay (ICD) [21], is expected to take place in an ultrashort femtosecond time scale [22], and also expected to occur at an interatomic distance of $\approx 3.76 \AA$ [23]. This is why it has been invoked for innershell or innervalence decays [23]. However, our study suggests a much shorter distance ( $\approx 1.7 \pm 0.2 \AA$ ), that seems to exclude this mechanism. On the other hand, the involvement of Rydberg states and large degrees of freedom as described in our scenario, favors curve crossing inducing autoionization as the most probable one in the case described here. Indeed at the distance found many electronic curve crossings can occur as it is the case in $\mathrm{Cl}_{2}$ [24] $(d=1.99 \AA)$, isoelectronic molecule of $\mathrm{Ar}_{2}^{2+}$.

In addition, scenario (iv) accounts for the fact that the recoil energy distribution of the smaller fragments is much broader as they recoil from all the $\mathrm{Ar}_{n \leq 50}^{+}$possible ions, which results in the same number of overlapping rings of slightly different energies. We notice that the rise with the average cluster size of the asymmetrical fragmentation channel has been observed by Rühl et al. [8] by using a soft-x-ray synchrotron radiation beam.

To summarize, we have analyzed surprising observations where $\operatorname{Ar}_{\bar{N}}$ clusters are ionized at moderate laser intensities. We have measured the recoil velocities of fragments $\mathrm{Ar}_{n=6 . \ldots 50}^{+}$resulting from a Coulomb explosion taking place into $\operatorname{Ar}_{500 \leqslant \bar{N} \leqslant 800}$ clusters, in the presence of Ti:sapphire laser pulses of moderate intensity $I_{L}=3.6 \times$ $10^{13} \mathrm{~W} \mathrm{~cm}^{-2}$. With the help of a VMI technique, we have evidenced the presence of remarkably well-resolved isotropic rings in the velocity distribution of these fragments. The analysis of the results points to a mechanism that involves the formation of a valence shell excited charged ion, followed by relaxation and charge transfer by autoionizing collision at very short distance. This last effect induces a Coulomb explosion followed by the fission of the cluster into two asymmetrical fragments, the distribution of the rings being associated to the recoil of smaller species $\operatorname{Ar}_{p=1,2,3,4}^{+}$. This phenomenon, which seems to be highly selective as it does not compete with other fragmentation channels, might be transferable.

The authors are happy to thank the laser team running the femtosecond laser facility LUCA of the CEA, DSM/ DRECAM, and Paul Morin (SOLEIL, Saint-Aubin, France) for fruitful discussions. We acknowledge financial support by Agence Nationale de la Recherche under Contract No. ANR-05-BLAN-0295-01, ATTO-SCIENCE.

*lionel.poisson@cea.fr

[1] U. Saalmann, Ch. Siedschlag, and J. M. Rost, J. Phys. B 39, R39 (2006).

[2] M. Lezius et al., Phys. Rev. Lett. 80, 261 (1998).

[3] T. Ditmire et al., Phys. Rev. A 57, 369 (1998).

[4] C. Deiss et al., Phys. Rev. Lett. 96, 013203 (2006).

[5] M. Lezius and T.D. Märk, Chem. Phys. Lett. 155, 496 (1989).

[6] W. Tappe et al., Phys. Rev. Lett. 88, 143401 (2002).

[7] E. Rühl, Int. J. Mass Spectrom. 229, 117 (2003).

[8] E. Rühl et al., Z. Phys. D 31, 245 (1994).

[9] M. Gisselbrecht et al., J. Chem. Phys. 123, 194301 (2005).

[10] N. T. Form et al., Phys. Chem. Chem. Phys. 8, 2925 (2006).

[11] J. M. Mestdagh et al., Int. Rev. Phys. Chem. 16, 215 (1997).

[12] A. Eppink and D. H. Parker, Rev. Sci. Instrum. 68, 3477 (1997).

[13] B. J. Whitaker, Imaging in Molecular Dynamics: Technology and Applications (Cambridge University Press, Cambridge, U.K., 2003).

[14] H. Haberland, Surf. Sci. 156, 305 (1985).

[15] D.J. Wales et al., http://www-wales.ch.cam.ac.uk/ CCD.html.

[16] S. Larochelle, A. Talebpour, and S. L. Chin, J. Phys. B 31, 1201 (1998).

[17] N. Saito et al., Chem. Phys. Lett. 441, 16 (2007).

[18] A. De Fanis et al., J. Phys. B 37, L235 (2004).

[19] C. Cachoncinlle et al., J. Chem. Phys. 96, 6085 (1992).

[20] J. M. Mestdagh et al. (to be published).

[21] L. S. Cederbaum, J. Zobeley, and F. Tarantelli, Phys. Rev. Lett. 79, 4778 (1997).

[22] A. I. Kuleff and L.S. Cederbaum, Phys. Rev. Lett. 98, 083201 (2007).

[23] Y. Morishita et al., Phys. Rev. Lett. 96, 243402 (2006).

[24] T. Möller et al., Chem. Phys. 76, 295 (1983). 